\title{
On some geometric properties for the combination of generalized Lommel-Wright function
}

\author{
Hanaa M. Zayed ${ }^{1 *}$ and Teodor Bulboacă
}

${ }^{\text {*Correspondence: }}$
hanaa@science.menofia.edu.eg
${ }^{1}$ Department of Mathematics and
Computer Science, Faculty of
Science, Menoufia University,
Shebin Elkom 32511, Egypt
Full list of author information is
available at the end of the article

available at the end of the article

\begin{abstract}
The scope of our investigation is to study the geometric properties of the normalized form of the combination of generalized Lommel-Wright function $J_{v, \lambda}^{\mu, m}$ defined by $\mathfrak{J}_{v, \lambda}^{\mu, m}(z):=\Gamma^{m}(\lambda+1) \Gamma(\lambda+v+1) 2^{2 \lambda+v} z^{1-(v / 2)-\lambda} \mathcal{I}_{v, \lambda}^{\mu, m}(\sqrt{z})$, where $\mathcal{I}_{\nu, \lambda}^{\mu, m}(z):=(1-2 \lambda-v) J_{\nu, \lambda}^{\mu, m}(z)+z\left(J_{v, \lambda}^{\mu, m}(z)\right)^{\prime}$ and

$$
J_{v, \lambda}^{\mu, m}(z)=\left(\frac{z}{2}\right)^{2 \lambda+v} \sum_{n=0}^{\infty} \frac{(-1)^{n}}{\Gamma^{m}(n+\lambda+1) \Gamma(n \mu+v+\lambda+1)}\left(\frac{z}{2}\right)^{2 n}
$$

with $m \in \mathbb{N}, \mu>0$ and $\lambda, v \in \mathbb{C}$, including starlikeness and convexity of order $\alpha$ $(0 \leq \alpha<1)$ in the open unit disc using the two-sided inequality for the Fox-Wright functions that has been proved by Pogány and Srivastava in (Comput. Math. Appl. 57(1):127-140, 2009). Further, the orders of starlikeness and convexity are also evaluated using some classical tools. We then compare the orders of starlikeness and convexity given by both techniques to illustrate the efficacy of the approach. In addition, we proved that for some values of $\alpha$, if $\lambda>-1$ then $\operatorname{Re}\left(\mathfrak{J}_{v, \lambda}^{\mu, m}(z) / z\right)>\alpha, z \in \mathbb{U}$, and if $\lambda \geq(\sqrt{10}-6) / 4$ then the function $\left(\mathfrak{J}_{v, \lambda}^{\mu, m}\left(z^{2}\right) / z\right) * \sin z$ is close-to-convex with respect to $1 / 2 \log ((1+z) /(1-z))$ where $*$ stands for the Hadamard product (or convolution) of two power series.
\end{abstract}

MSC: $30 C 45 ; 30 C 50$

Keywords: Analytic; Univalent; Starlike; Convex; Close-to-convex; Generalized Lommel-Wright functions

\section{Introduction and preliminaries}

Special functions have attracted the particular attention of researchers due to their applications in many branches of mathematics and physics. Recently, special attention has further been devoted by several mathematicians to study the geometric properties of special functions like starlikeness, convexity, and close-to-convexity in the open unit disc. One can see the following papers in this direction: for hypergeometric functions $([6,7]$ and [12, 13]), Bessel functions [1, 2], generalized Struve functions [8, 17], and Lommel

(c) The Author(s) 2021. This article is licensed under a Creative Commons Attribution 4.0 International License, which permits use, sharing, adaptation, distribution and reproduction in any medium or format, as long as you give appropriate credit to the original author(s) and the source, provide a link to the Creative Commons licence, and indicate if changes were made. The images or other third party material in this article are included in the article's Creative Commons licence, unless indicated otherwise in a credit line to the material. If material is not included in the article's Creative Commons licence and your intended use is not permitted by statutory regulation or exceeds the permitted use, you will need to obtain permission directly from the copyright holder. To view a copy of this licence, visit http://creativecommons.org/licenses/by/4.0/. 
functions [16]. Results of this type seem to be more and more visible in the developments of Geometric Function Theory. Their analyses are not only auxiliary tools but also provide powerful information to generalize to several types of functions and polynomials.

It is worthy of mention that generalized Lommel-Wright function have been constructed from concrete problems in mechanics, physics, engineering, and astronomy. Our main results are summarized in the following: we study some geometric properties for the normalized form of the combination of generalized Lommel-Wright function and its derivative, including starlikeness and convexity of order $\alpha(0 \leq \alpha<1)$ in the open unit $\operatorname{disc} \mathbb{U}:=\{z \in \mathbb{C}:|z|<1\}$ using the two-sided inequality for the Fox-Wright functions that has been proved by Pogány and Srivastava in [11]. In addition, the orders of starlikeness and convexity are also established using some classical tools. We then compare the orders of starlikeness and convexity given by both techniques to illustrate the efficacy of the approach.

For all of what follows, let $\mathcal{H}$ denote the space of all analytic functions in $\mathbb{U}$. Denote by $\mathcal{A}$ the subclass of $\mathcal{H}$ of functions of the form

$$
f(z)=z+\sum_{n=2}^{\infty} \mathrm{A}_{n} z^{n}, \quad z \in \mathbb{U}
$$

and by $\mathcal{S}$ the subclass of all functions in $\mathcal{A}$ that are univalent in $\mathbb{U}$. If $g \in \mathcal{A}$ is given by

$$
g(z)=z+\sum_{n=2}^{\infty} \mathrm{B}_{n} z^{n}, \quad z \in \mathbb{U}
$$

then the Hadamard product (or convolution) of $f$ and $g$ is defined by

$$
(f * g)(z):=z+\sum_{n=2}^{\infty} \mathrm{A}_{n} \mathrm{~B}_{n} z^{n}=(g * f)(z), \quad z \in \mathbb{U}
$$

For $0 \leq \alpha<1$, a function $f \in \mathcal{A}$ is said to be in the class of starlike functions of order $\alpha$, denoted by $\mathcal{S}^{*}(\alpha)$, if and only if $\operatorname{Re}\left(z f^{\prime}(z) / f(z)\right)>\alpha$, for all $z \in \mathbb{U}$, and is said to be in the class of convex functions of order $\alpha$, denoted by $\mathcal{K}(\alpha)$, if and only if $1+\operatorname{Re}\left(z f^{\prime \prime}(z) / f^{\prime}(z)\right)>\alpha$, for all $z \in \mathbb{U}$. Also, $f \in \mathcal{H}$ is said to be a close-to-convex function with respect to a fixed starlike function $g \in \mathcal{S}^{*}(0)$ (which need not be normalized), denoted by $\mathcal{C}_{g}$, if and only if $\operatorname{Re}\left(z f^{\prime}(z) / g(z)\right)>0, z \in \mathbb{U}$. It is well known that $\mathcal{K}(\alpha) \subset \mathcal{S}^{*}(\alpha)$, and all these three subclasses of functions, namely $\mathcal{S}^{*}(\alpha), \mathcal{K}(\alpha)$, and $\mathcal{C}_{g}$, are subsets of $\mathcal{S}$. In addition, for $\alpha<1$, let recall the class

$$
\mathcal{P}(\alpha):=\{p \in \mathcal{H}: p(0)=1, \operatorname{Re} p(z)>\alpha, z \in \mathbb{U}\} .
$$

The generalized Lommel-Wright function $J_{v, \lambda}^{\mu, m}(z)$ is defined by de Oteiza et al. in [3] as

$$
\begin{aligned}
J_{v, \lambda}^{\mu, m}(z) & =\left(\frac{z}{2}\right)^{2 \lambda+v} \sum_{n=0}^{\infty} \frac{(-1)^{n}}{\Gamma^{m}(n+\lambda+1) \Gamma(n \mu+v+\lambda+1)}\left(\frac{z}{2}\right)^{2 n} \\
& =\left(\frac{z}{2}\right)^{2 \lambda+v}{ }_{1} \Psi_{m+1}\left[\begin{array}{c}
(1,1) \\
(\lambda+1,1), \ldots,(\lambda+1,1),(\lambda+v+1, \mu)
\end{array} \mid-\frac{z^{2}}{4}\right],
\end{aligned}
$$


for $m \in \mathbb{N}:=\{1,2, \ldots\}, \mu>0$ and $\lambda, v \in \mathbb{C}$ where $\Psi_{q}$ denotes the Fox-Wright generalization of the hypergeometric function which is defined by

$$
\left.\left.{ }_{p} \Psi_{q}\left[\begin{array}{c}
\left(a_{1}, A_{1}\right), \ldots,\left(a_{p}, A_{p}\right) \\
\left(b_{1}, B_{1}\right), \ldots,\left(b_{q}, B_{q}\right)
\end{array}\right) z\right]=: \Psi_{p} \Psi_{q}\left[\begin{array}{c}
\left(\mathbf{a}_{p}, \mathbf{A}_{p}\right) \\
\left(\mathbf{b}_{q}, \mathbf{B}_{q}\right)
\end{array}\right) z\right]=\sum_{n=0}^{\infty} \psi_{n} \frac{z^{n}}{n !},
$$

with

$$
\psi_{n}=\frac{\Gamma\left(a_{1}+A_{1} n\right) \cdots \Gamma\left(a_{p}+A_{p} n\right)}{\Gamma\left(b_{1}+B_{1} n\right) \cdots \Gamma\left(b_{q}+B_{q} n\right)},
$$

for $a_{i}, b_{j} \in \mathbb{C}$ and $A_{i}, B_{j} \in \mathbb{R}^{+}(i=1, \ldots, p, j=1, \ldots, q)$. It is worth noting that the above series converges absolutely in the whole complex $z$-plane when $\Delta:=\sum_{j=1}^{q} B_{j}-\sum_{i=1}^{p} A_{i}>$ -1 , while if $\Delta=-1$, then the series (1.3) converges absolutely for $|z|<\rho$ and $|z|=\rho$ under the condition $\operatorname{Re}(\sigma)>1 / 2$ where

$$
\rho=\left(\prod_{i=1}^{p} A_{i}^{-A_{i}}\right)\left(\prod_{j=1}^{q} B_{j}^{-B_{j}}\right), \quad \sigma=\sum_{j=1}^{q} b_{j}-\sum_{i=1}^{p} a_{i}+\frac{p-q}{2} .
$$

We refer the interested readers to [5] for more details concerning the Fox-Wright functions.

As a special case of the generalized Lommel-Wright function (1.2) obtained by taking $m=1$ in (1.2), we obtain the following generalization of the Bessel function introduced by Pathak [10]:

$$
J_{v, \lambda}^{\mu}(z):=J_{v, \lambda}^{\mu, 1}(z)=\left(\frac{z}{2}\right)^{2 \lambda+\nu} \sum_{n=0}^{\infty} \frac{(-1)^{n}}{\Gamma(n+\lambda+1) \Gamma(n \mu+v+\lambda+1)}\left(\frac{z}{2}\right)^{2 n},
$$

for $\mu>0$ and $\lambda, v \in \mathbb{C}$. If $m=1, \mu=1$ and $\lambda=1 / 2$, we obtain the Struve function defined by the power series expansion

$$
H_{\nu}(z):=J_{v, 1 / 2}^{1,1}(z)=\sum_{n=0}^{\infty} \frac{(-1)^{n}}{\Gamma(n+3 / 2) \Gamma(n+v+3 / 2)}\left(\frac{z}{2}\right)^{v+2 n}, \quad v \in \mathbb{C} .
$$

For $m=1, \mu=1$, and $\lambda=0$ in (1.2), we get the Bessel function defined by

$$
J_{v}(z):=J_{v, 1 / 2}^{1,1}(z)=\sum_{n=0}^{\infty} \frac{(-1)^{n}}{n ! \Gamma(n+v+1)}\left(\frac{z}{2}\right)^{v+2 n},
$$

where $z, v \in \mathbb{C}, z \neq 0$ and $\operatorname{Re} v>-1$.

We shall base our discussion upon the following definition:

Definition 1.1 Let $m \in \mathbb{N}, \lambda \in \mathbb{C} \backslash \mathbb{Z}^{-}$with $\mathbb{Z}^{-}:=\{-1,-2,-3, \ldots\}$ and $\nu, \mu \in \mathbb{N}_{0}:=\mathbb{N} \cup\{0\}$. We define the normalized form of the combination of generalized Lommel-Wright function by

$$
\mathfrak{J}_{v, \lambda}^{\mu, m}(z):=\Gamma^{m}(\lambda+1) \Gamma(\lambda+v+1) 2^{2 \lambda+v} z^{1-(v / 2)-\lambda} \mathcal{I}_{v, \lambda}^{\mu, m}(\sqrt{z})
$$

where $\mathcal{I}_{v, \lambda}^{\mu, m}(z):=(1-2 \lambda-v) J_{v, \lambda}^{\mu, m}(z)+z\left(J_{v, \lambda}^{\mu, m}(z)\right)^{\prime}$. 
It can further be shown that

$$
\mathfrak{J}_{v, \lambda}^{\mu, m}(z)=z+\sum_{n=1}^{\infty} \frac{(-1)^{n}(2 n+1)}{4^{n}\left[(\lambda+1)_{n}\right]^{m}(\lambda+v+1)_{n \mu}} z^{n+1}
$$

where $(a)_{n}$ represents the Pochhammer symbol defined by

$$
(a)_{n}:= \begin{cases}1, & \text { if } n=0, \\ a(a+1)(a+2) \cdots(a+n-1), & \text { if } n \in \mathbb{N} .\end{cases}
$$

To establish our results, we need the following two lemma.

The first is a classical result of Fejér [4] and may be found in the next form in [14, Theorem 2.10]:

Lemma 1.1 Assume $\mathrm{A}_{1}=1$ and $\mathrm{A}_{n} \geq 0$ for $n \geq 2$ such that $\left(\mathrm{A}_{n}\right)_{n \in \mathbb{N}}$ is a convex decreasing sequence, that is, $\mathrm{A}_{n}-2 \mathrm{~A}_{n+1}+\mathrm{A}_{n+2} \geq 0$ and $\mathrm{A}_{n+1}-\mathrm{A}_{n+2} \geq 0$, for all $n \in \mathbb{N}$. Then, $\operatorname{Re}\left(\sum_{n=1}^{\infty} \mathrm{A}_{n} z^{n-1}\right)>1 / 2, z \in \mathbb{U}$.

The next lemma is a special case of [9, Corollary 9] (see also [9, Theorem 10]) for the odd functions. It follows since the hypothesis ensures that $f$ is close-to-convex function with respect to $\log \sqrt{(1+z) /(1-z)}$.

Lemma 1.2 Suppose that the function $f$ defined by (1.1) is an odd function (that is, $\mathrm{A}_{2 n}=$ 0 for all $n \in \mathbb{N})$, such that $1 \geq 3 \mathrm{~A}_{3} \geq \cdots \geq(2 n+1) \mathrm{A}_{2 n+1} \geq 0$, or $1 \leq 3 \mathrm{~A}_{3} \leq \cdots \leq(2 n+$ 1) $\mathrm{A}_{2 n+1} \leq 2$. Then $f \in \mathcal{S}$.

\section{Main results}

Theorem 2.1 Suppose that $\lambda>0$ and $\nu, \mu \in \mathbb{N}$ satisfy the inequalities

$$
\begin{aligned}
& \frac{1}{\Gamma(2 \mu+\lambda+v+1)}>\frac{21}{20(\lambda+3)^{m} \Gamma(3 \mu+\lambda+v+1)}, \\
& \frac{21}{20(\lambda+3)^{m} \Gamma(\mu+\lambda+v+1) \Gamma(3 \mu+\lambda+v+1)} \\
& >\frac{5}{6(\lambda+2)^{m} \Gamma^{2}(2 \mu+\lambda+v+1)} .
\end{aligned}
$$

If

$$
\begin{aligned}
0 \leq & \alpha \\
\leq & 1-\frac{3 \Gamma(\lambda+v+1)}{4(\lambda+1)^{m}} \cdot \frac{(\lambda+1)^{m}(\lambda+v+1)^{\mu}-1}{(\lambda+1)^{m}(\lambda+v+1)^{\mu}-2} \\
& \cdot\left[\frac{1}{\Gamma(\lambda+v+\mu+1)}-\frac{5(1-e)}{6(\lambda+2)^{m} \Gamma(\lambda+v+2 \mu+1)}\right] \\
= & : \widetilde{\alpha}_{\max },
\end{aligned}
$$

then $\mathfrak{J}_{v, \lambda}^{\mu, m} \in \mathcal{S}^{*}(\alpha)$. 
Proof To obtain our result $\mathfrak{J}_{v, \lambda}^{\mu, m} \in \mathcal{S}^{*}(\alpha)$, it is sufficient to prove that

$$
\left|\frac{z\left(\mathfrak{J}_{v, \lambda}^{\mu, m}(z)\right)^{\prime}}{\mathfrak{J}_{v, \lambda}^{\mu, m}(z)}-1\right|<1-\alpha, \quad z \in \mathbb{U}
$$

Using the theorem of the maximum of the module of an analytic function and the triangle inequality, a simple computation shows that

$$
\begin{aligned}
& \left|\frac{z\left(\mathfrak{J}_{v, \lambda}^{\mu, m}(z)\right)^{\prime}}{\mathfrak{J}_{v, \lambda}^{\mu, m}(z)}-1\right|=\left|\frac{\sum_{n=1}^{\infty} \frac{(-1)^{n} n(2 n+1)}{4^{n}\left[(\lambda+1)_{n}\right]^{m}(\lambda+v+1)_{n \mu}} z^{n+1}}{z+\sum_{n=1}^{\infty} \frac{(-1)^{n}(2 n+1)}{4^{n}\left[(\lambda+1)_{n}\right]^{m}(\lambda+v+1)_{n \mu}} z^{n+1}}\right| \\
& =\frac{\left|\sum_{n=1}^{\infty} \frac{(-1)^{n} n(2 n+1)}{4^{n}\left[(\lambda+1)_{n}\right]^{m}(\lambda+v+1)_{n \mu}} z^{n}\right|}{\left|1+\sum_{n=1}^{\infty} \frac{(-1)^{n}(2 n+1)}{4^{n}\left[(\lambda+1)_{n}\right]^{m}(\lambda+\nu+1)_{n \mu}} z^{n}\right|} \\
& <\frac{\sum_{n=1}^{\infty} \frac{n(2 n+1)}{4^{n}\left[(\lambda+1)_{n}\right]^{m}(\lambda+v+1)_{n \mu}}}{1-\sum_{n=1}^{\infty} \frac{2 n+1}{4^{n}\left[(\lambda+1)_{n}\right]^{m}(\lambda+v+1)_{n \mu}}}=: \frac{\mathrm{F}_{1}}{\mathrm{~F}_{2}}, \quad z \in \mathbb{U} .
\end{aligned}
$$

First, we see that

$$
\begin{aligned}
\mathrm{F}_{1} & =\sum_{n=1}^{\infty} \frac{n(2 n+1)}{4^{n}\left[(\lambda+1)_{n}\right]^{m}(\lambda+v+1)_{n \mu}} \frac{(1)_{n}(2)_{2 n}}{(1)_{n}(2)_{2 n}} \\
& =\sum_{n=1}^{\infty} \frac{1}{4^{n}\left[(\lambda+1)_{n}\right]^{m}(\lambda+v+1)_{n \mu}} \frac{(1)_{n}(2)_{2 n}}{(1)_{n-1}(1)_{2 n}}, \quad n \in \mathbb{N} .
\end{aligned}
$$

Using the Legendre's formula

$$
\Gamma(z) \Gamma\left(z+\frac{1}{2}\right)=\frac{\sqrt{\pi}}{2^{2 z-1}} \Gamma(2 z),
$$

for $z=n+1$ we get

$$
\Gamma\left(n+\frac{3}{2}\right)=\frac{1 \cdot 3 \cdots(2 n+1)}{2^{n+1}} \sqrt{\pi}, \quad n \in \mathbb{N},
$$

and also

$$
(2)_{2 n}=4^{n}(1)_{n}\left(\frac{3}{2}\right)_{n}, \quad n \in \mathbb{N} .
$$

From the identities (2.5) and (2.6), it follows that

$$
\begin{aligned}
\sum_{n=1}^{\infty} & \frac{1}{4^{n}\left[(\lambda+1)_{n}\right]^{m}(\lambda+v+1)_{n \mu}} \frac{(1)_{n}(2)_{2 n}}{(1)_{n-1}(1)_{2 n}} \\
= & \frac{2 \Gamma^{m}(\lambda+1) \Gamma(\lambda+v+1)}{\sqrt{\pi}} \\
& \cdot \sum_{n=0}^{\infty} \frac{\Gamma(n+2) \Gamma(n+2) \Gamma(n+5 / 2)}{\Gamma^{m}(n+\lambda+2) \Gamma(n \mu+\mu+\lambda+v+1) \Gamma(n+1) \Gamma(2 n+3)}
\end{aligned}
$$




$$
\begin{aligned}
= & \frac{2 \Gamma^{m}(\lambda+1) \Gamma(\lambda+v+1)}{\sqrt{\pi}} \\
& \cdot{ }_{3} \Psi_{m+2}\left[\begin{array}{c}
(2,1),(2,1),(5 / 2,1) \\
(\lambda+2,1), \ldots,(\lambda+2,1),(\lambda+v+\mu+1, \mu),(3,2)
\end{array} \mid 1\right] .
\end{aligned}
$$

On the other hand, according to Theorem 4 of [11], the next inequality holds for all ${ }_{p} \Psi_{q}$ satisfying $\psi_{1}>\psi_{2}$ and $\psi_{1}^{2}<\psi_{0} \psi_{2}$ :

$$
\psi_{0} e^{\psi_{1} \psi_{0}^{-1}|z|} \leq{ }_{p} \Psi_{q}\left[\begin{array}{c}
\left(\mathbf{a}_{p}, \mathbf{A}_{p}\right) \\
\left(\mathbf{b}_{q}, \mathbf{B}_{q}\right)
\end{array} \mid z\right] \leq \psi_{0}-\left(1-e^{|z|}\right) \psi_{1}, \quad z \in \mathbb{R} .
$$

For the Fox-Wright function that appears in (2.7), we have

$$
\psi_{n}=\frac{\Gamma(n+2) \Gamma(n+2) \Gamma(n+5 / 2)}{\Gamma^{m}(n+\lambda+2) \Gamma(n \mu+\mu+\lambda+v+1) \Gamma(2 n+3)},
$$

hence

$$
\begin{aligned}
& \psi_{0}=\frac{\Gamma(2) \Gamma(2) \Gamma(5 / 2)}{\Gamma^{m}(\lambda+2) \Gamma(\mu+\lambda+v+1) \Gamma(3)}, \\
& \psi_{1}=\frac{\Gamma(3) \Gamma(3) \Gamma(7 / 2)}{\Gamma^{m}(\lambda+3) \Gamma(2 \mu+\lambda+v+1) \Gamma(5)},
\end{aligned}
$$

and

$$
\psi_{2}=\frac{\Gamma(4) \Gamma(4) \Gamma(9 / 2)}{\Gamma^{m}(\lambda+4) \Gamma(3 \mu+\lambda+v+1) \Gamma(7)} .
$$

It is easy to verify that the inequalities $\psi_{1}>\psi_{2}$ and $\psi_{1}^{2}<\psi_{0} \psi_{2}$ are equivalent to the assumptions (2.1) and (2.2), respectively. Hence, according to the right-hand side of the double inequality (2.8), from (2.7) we obtain

$$
\mathrm{F}_{1} \leq \frac{3 \Gamma(\lambda+v+1)}{4(\lambda+1)^{m}}\left[\frac{1}{\Gamma(\lambda+v+\mu+1)}-\frac{5(1-e)}{6(\lambda+2)^{m} \Gamma(\lambda+v+2 \mu+1)}\right] .
$$

Moreover, since

$$
{ }_{3} \Psi_{m+2}\left[\begin{array}{c|c}
(2,1),(2,1),(5 / 2,1) \\
(\lambda+2,1), \ldots,(\lambda+2,1),(\lambda+v+\mu+1, \mu),(3,2)
\end{array} \mid 1\right]>0
$$

for $\lambda>0$ and $v, \mu \in \mathbb{N}$, it follows that the right-hand side of the inequality (2.9) is positive.

Secondly, using the fact that $2 n+1 \leq 4^{n}, n \in \mathbb{N}$, and under our assumptions

$$
(\lambda+v+1)_{n \mu} \geq(\lambda+v+1)^{n \mu}
$$

we have

$$
\mathrm{F}_{2}=1-\sum_{n=1}^{\infty} \frac{2 n+1}{4^{n}\left[(\lambda+1)_{n}\right]^{m}(\lambda+v+1)_{n \mu}}
$$




$$
\begin{aligned}
& \geq 1-\sum_{n=1}^{\infty}\left(\frac{1}{(\lambda+1)^{m}(\lambda+v+1)^{\mu}}\right)^{n} \\
& =\frac{(\lambda+1)^{m}(\lambda+v+1)^{\mu}-2}{(\lambda+1)^{m}(\lambda+v+1)^{\mu}-1}>0, \quad \text { for } \lambda>0 \text { and } v, \mu \in \mathbb{N}
\end{aligned}
$$

Using (2.9) and (2.11), from the inequality (2.4) we deduce that

$$
\begin{aligned}
\left|\frac{z\left(\mathfrak{J}_{v, \lambda}^{\mu, m}(z)\right)^{\prime}}{\mathfrak{J}_{v, \lambda}^{\mu, m}(z)}-1\right| & <\frac{\mathrm{F}_{1}}{\mathrm{~F}_{2}} \\
& \leq \frac{\frac{3 \Gamma(\lambda+v+1)}{4(\lambda+1)^{m}}\left[\frac{1}{\Gamma(\lambda+v+\mu+1)}-\frac{5(1-e)}{6(\lambda+2)^{m} \Gamma(\lambda+v+2 \mu+1)}\right]}{\frac{(\lambda+1)^{m}(\lambda+v+1)^{\mu}-2}{(\lambda+1)^{m}(\lambda+v+1)^{\mu}-1}} \\
& \leq 1-\alpha, \quad z \in \mathbb{U},
\end{aligned}
$$

whenever the assumption (2.3) holds, and the theorem is completely proved.

Theorem 2.2 Suppose that $\lambda>0$ and $\nu, \mu \in \mathbb{N}$ satisfy the inequalities

$$
\begin{aligned}
& \frac{1}{\Gamma(2 \mu+\lambda+v+1)}>\frac{7}{5(\lambda+3)^{m} \Gamma(3 \mu+\lambda+v+1)}, \\
& \frac{7}{5(\lambda+3)^{m} \Gamma(\mu+\lambda+v+1) \Gamma(3 \mu+\lambda+v+1)} \\
& >\frac{5}{4(\lambda+2)^{m} \Gamma^{2}(2 \mu+\lambda+v+1)}, \\
& 2(\lambda+1)^{m}(\lambda+v+1)^{\mu}>5 .
\end{aligned}
$$

If

$$
\begin{aligned}
0 \leq & \alpha \\
\leq & 1-\frac{(\lambda+1)^{m}(\lambda+v+1)^{\mu}-1}{2(\lambda+1)^{m}(\lambda+v+1)^{\mu}-5} \cdot \frac{3 \Gamma(\lambda+v+1)}{(\lambda+1)^{m}} \\
& \cdot\left[\frac{1}{\Gamma(\lambda+v+\mu+1)}-\frac{5(1-e)}{4(\lambda+2)^{m} \Gamma(\lambda+v+2 \mu+1)}\right] \\
= & : \widetilde{\beta}_{\max },
\end{aligned}
$$

then $\mathfrak{J}_{v, \lambda}^{\mu, m} \in \mathcal{K}(\alpha)$

Proof A sufficient condition for the function $\mathfrak{J}_{v, \lambda}^{\mu, m}$ to be convex of order $\alpha$ is to prove that

$$
\left|\frac{z\left(\mathfrak{J}_{v, \lambda}^{\mu, m}(z)\right)^{\prime \prime}}{\left(\mathfrak{J}_{v, \lambda}^{\mu, m}(z)\right)^{\prime}}\right|<1-\alpha, \quad z \in \mathbb{U}
$$


Like in the proof of the previous theorem, using the theorem of the maximum of the module of an analytic function and the triangle inequality, we find

$$
\left|\frac{z\left(\mathfrak{J}_{v, \lambda}^{\mu, m}(z)\right)^{\prime \prime}}{\left(\mathfrak{J}_{v, \lambda}^{\mu, m}(z)\right)^{\prime}}\right|<\frac{\sum_{n=1}^{\infty} \frac{n(n+1)(2 n+1)}{4^{n}\left[(\lambda+1)_{n}\right]^{m}(\lambda+\nu+1)_{n \mu}}}{1-\sum_{n=1}^{\infty} \frac{(n+1)(2 n+1)}{4^{n}\left[(\lambda+1)_{n}\right]^{m}(\lambda+v+1)_{n \mu}}}=: \frac{\mathrm{G}_{1}}{\mathrm{G}_{2}} .
$$

Like in the proof of the Theorem 2.1, from the identities (2.5) and (2.6), we have

$$
\begin{aligned}
\mathrm{G}_{1}= & \sum_{n=1}^{\infty} \frac{n(n+1)(2 n+1)}{4^{n}\left[(\lambda+1)_{n}\right]^{m}(\lambda+v+1)_{n \mu}} \frac{(2)_{n}(1)_{n}(2)_{2 n}}{(2)_{n}(1)_{n}(2)_{2 n}} \\
= & \sum_{n=1}^{\infty} \frac{1}{4^{n}\left[(\lambda+1)_{n}\right]^{m}(\lambda+v+1)_{n \mu}} \frac{(2)_{n}(1)_{n}(2)_{2 n}}{(1)_{n}(1)_{n-1}(1)_{2 n}} \\
= & \frac{2 \Gamma^{m}(\lambda+1) \Gamma(\lambda+v+1)}{\sqrt{\pi}} \\
& \cdot \sum_{n=0}^{\infty} \frac{\Gamma(n+3) \Gamma(n+2) \Gamma(n+5 / 2)}{\Gamma^{m}(n+\lambda+2) \Gamma(n \mu+\mu+\lambda+v+1) \Gamma(n+1) \Gamma(2 n+3)} \\
= & \frac{2 \Gamma^{m}(\lambda+1) \Gamma(\lambda+v+1)}{\sqrt{\pi}} \quad(3,1),(2,1),(5 / 2,1) \\
& \cdot{ }_{3} \Psi_{m+2}[(\lambda+2,1), \ldots,(\lambda+2,1),(\lambda+v+\mu+1, \mu),(3,2) \mid 1] .
\end{aligned}
$$

For the above Fox-Wright function (2.17), we have

$$
\psi_{n}=\frac{\Gamma(n+3) \Gamma(n+2) \Gamma(n+5 / 2)}{\Gamma^{m}(n+\lambda+2) \Gamma(n \mu+\mu+\lambda+v+1) \Gamma(2 n+3)},
$$

hence

$$
\begin{aligned}
& \psi_{0}=\frac{\Gamma(3) \Gamma(2) \Gamma(5 / 2)}{\Gamma^{m}(\lambda+2) \Gamma(\mu+\lambda+v+1) \Gamma(3)}, \\
& \psi_{1}=\frac{\Gamma(4) \Gamma(3) \Gamma(7 / 2)}{\Gamma^{m}(\lambda+3) \Gamma(2 \mu+\lambda+v+1) \Gamma(5)},
\end{aligned}
$$

and

$$
\psi_{2}=\frac{\Gamma(5) \Gamma(4) \Gamma(9 / 2)}{\Gamma^{m}(\lambda+4) \Gamma(3 \mu+\lambda+v+1) \Gamma(7)} .
$$

We could easily check that the inequalities $\psi_{1}>\psi_{2}$ and $\psi_{1}^{2}<\psi_{0} \psi_{2}$ are equivalent to the assumptions (2.12) and (2.13), respectively. Using again the right-hand side of the double inequality (2.8), from (2.17) we have

$$
\mathrm{G}_{1} \leq \frac{3 \Gamma^{m}(\lambda+1) \Gamma(\lambda+v+1)}{2 \Gamma^{m}(\lambda+2)}\left[\frac{1}{\Gamma(\lambda+v+\mu+1)}-\frac{5(1-e)}{4(\lambda+2)^{m} \Gamma(\lambda+v+2 \mu+1)}\right]
$$


Also, since

$$
{ }_{3} \Psi_{m+2}\left[\begin{array}{c|c}
(3,1),(2,1),(5 / 2,1) \\
(\lambda+2,1), \ldots,(\lambda+2,1),(\lambda+v+\mu+1, \mu),(3,2)
\end{array} \mid 1\right]>0
$$

for $\lambda>0$ and $\nu, \mu \in \mathbb{N}$, it follows that the right-hand side of the inequality (2.18) is positive.

Furthermore, since $(n+1)(2 n+1) \leq(3 / 2) 4^{n}, n \in \mathbb{N}$, using (2.10), we obtain

$$
\begin{aligned}
\mathrm{G}_{2} & =1-\sum_{n=1}^{\infty} \frac{(n+1)(2 n+1)}{4^{n}\left[(\lambda+1)_{n}\right]^{m}(\lambda+v+1)_{n \mu}} \\
& \geq 1-\frac{3}{2} \sum_{n=1}^{\infty}\left(\frac{1}{(\lambda+1)^{m}(\lambda+v+1)^{\mu}}\right)^{n} \\
& =1-\frac{3}{2} \frac{1}{(\lambda+1)^{m}(\lambda+v+1)^{\mu}-1} \\
& =\frac{2(\lambda+1)^{m}(\lambda+v+1)^{\mu}-5}{2\left[(\lambda+1)^{m}(\lambda+v+1)^{\mu}-1\right]}>0, \quad \text { for } \lambda>0, v, \mu \in \mathbb{N},
\end{aligned}
$$

if the assumption (2.14) holds.

Using (2.18) and (2.19), inequality (2.16) leads to

$$
\begin{aligned}
&\left|\frac{z\left(\mathfrak{J}_{v, \lambda}^{\mu, m}(z)\right)^{\prime \prime}}{\left(\mathfrak{J}_{v, \lambda}^{\mu, m}(z)\right)^{\prime}}\right|<\frac{\mathrm{G}_{1}}{\mathrm{G}_{2}} \\
& \leq \frac{\frac{3 \Gamma(\lambda+v+1)}{4(\lambda+1)^{m}}\left[\frac{1}{\Gamma(\lambda+v+\mu+1)}-\frac{5(1-e)}{4(\lambda+2)^{m} \Gamma(\lambda+v+2 \mu+1)}\right]}{\frac{2(\lambda+1)^{m}(\lambda+v+1)^{\mu}-5}{2\left[(\lambda+1)^{m}(\lambda+v+1)^{\mu}-1\right]}} \\
& \leq 1-\alpha, \quad z \in \mathbb{U},
\end{aligned}
$$

whenever the assumption (2.15) holds, and our result is proved.

Remark 2.1 We emphasize that the assumptions of Theorems 2.1 and 2.2 can be simultaneously fulfilled, as it is shown in the below Fig. 1 (the grey regions) obtained by using the MAPLE $^{\text {mat }}$ software.

Theorem 2.3 Suppose that

$$
\begin{aligned}
0 & \leq \alpha \leq 1-\frac{3(\lambda+2)^{m}(\lambda+v+2)}{4(\lambda+1)^{m}(\lambda+v+1)\left[(\lambda+2)^{m}(\lambda+v+2)-1\right]-3(\lambda+2)^{m}(\lambda+v+2)} \\
& =: \widehat{\alpha}_{\max }
\end{aligned}
$$

with $\lambda>-1$ and $\nu, \mu \in \mathbb{N}$, which also satisfies

$$
\frac{4(\lambda+1)^{m}(\lambda+v+1)\left[(\lambda+2)^{m}(\lambda+v+2)-1\right]}{3(\lambda+2)^{m}(\lambda+v+2)}>1 .
$$

Then, $\mathfrak{J}_{v, \lambda}^{\mu, m} \in \mathcal{S}^{*}(\alpha)$. 


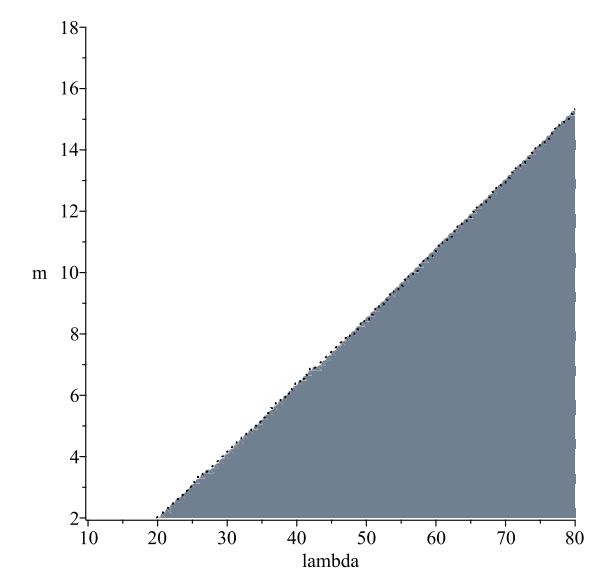

(A) The region where the assumptions (2.1),

(2.2) and (2.3) can be simultaneously fulfilled for $\mu=2, \nu=4$, with $\lambda \in[10,80]$ and $m \in[2,18]$

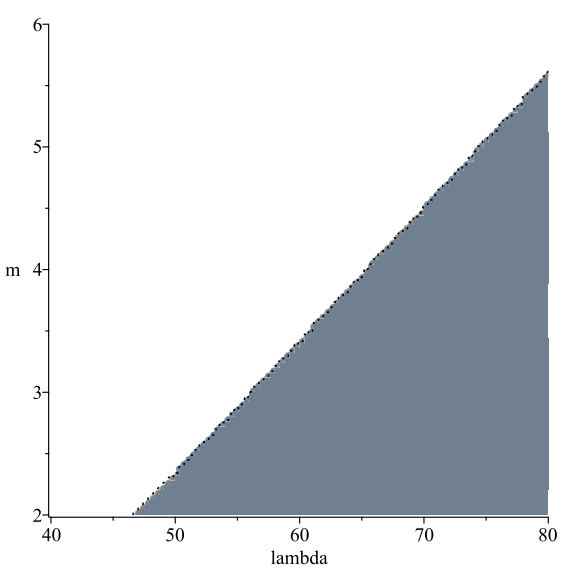

(B) The region where the assumptions (2.12), (2.13), (2.14) and (2.15) could be simultaneously fulfilled for $\mu=2, \nu=4$, with $\lambda \in[40,80]$ and $m \in[2,6]$

Figure 1 The figures for Remark 2.1

Proof It is well-known from [15, Theorem 1] that if $f$ of the form (1.1) satisfies $\sum_{n=2}^{\infty}(n-$ $\alpha)\left|A_{n}\right| \leq 1-\alpha$, then $f \in \mathcal{S}^{*}(\alpha)$. Thus, according to (1.4), it is enough to prove that

$$
\mathrm{H}_{1}:=\sum_{n=2}^{\infty}(n-\alpha)\left|\frac{(-1)^{n-1}(2 n-1)}{4^{n-1}\left[(\lambda+1)_{n-1}\right]^{m}(\lambda+v+1)_{(n-1) \mu}}\right| \leq 1-\alpha .
$$

Since $\lambda>-1$ and $\mu \in \mathbb{N}$, we have

$$
\begin{aligned}
\mathrm{H}_{1} & =\sum_{n=2}^{\infty} \frac{(n-\alpha)(2 n-1)}{4^{n-1}\left[(\lambda+1)_{n-1}\right]^{m}(\lambda+v+1)_{(n-1) \mu}} \\
& =\sum_{n=1}^{\infty} \frac{n(2 n+1)}{4^{n}\left[(\lambda+1)_{n}\right]^{m}(\lambda+v+1)_{n \mu}}+(1-\alpha) \sum_{n=1}^{\infty} \frac{2 n+1}{4^{n}\left[(\lambda+1)_{n}\right]^{m}(\lambda+v+1)_{n \mu}},
\end{aligned}
$$

and using the fact that

$$
(\lambda+v+1)_{n \mu} \geq(\lambda+v+1)_{n}, \quad \text { for all } n, v, \mu \in \mathbb{N},
$$

it follows that

$$
\begin{aligned}
\mathrm{H}_{1} \leq & \frac{1}{4(\lambda+1)^{m}(\lambda+v+1)} \sum_{n=1}^{\infty} \frac{n(2 n+1)}{4^{n-1}\left[(\lambda+2)_{n-1}\right]^{m}(\lambda+v+2)_{n-1}} \\
& +\frac{1-\alpha}{4(\lambda+1)^{m}(\lambda+v+1)} \sum_{n=1}^{\infty} \frac{2 n+1}{4^{n-1}\left[(\lambda+2)_{n-1}\right]^{m}(\lambda+v+2)_{n-1}} .
\end{aligned}
$$

Moreover, it is easy to check that

$$
n(2 n+1) \leq 3 \cdot 4^{n-1}, \quad 2 n+1 \leq 3 \cdot 4^{n-1},
$$




$$
(\lambda+v+2)_{n-1} \geq(\lambda+v+2)^{n-1}, \quad(\lambda+2)_{n-1} \geq(\lambda+2)^{n-1}
$$

for all $n \in \mathbb{N}$. From (2.23), using the above inequalities together with the assumptions (2.20) and (2.21), as well as the facts that $\lambda>-1$ and $v \in \mathbb{N}$, we get

$$
\begin{aligned}
\mathrm{H}_{1} \leq & \frac{3}{4(\lambda+1)^{m}(\lambda+v+1)} \sum_{n=1}^{\infty} \frac{1}{(\lambda+2)^{(n-1) m}(\lambda+v+2)^{n-1}} \\
& +\frac{3(1-\alpha)}{4(\lambda+1)^{m}(\lambda+v+1)} \sum_{n=1}^{\infty} \frac{1}{(\lambda+2)^{(n-1) m}(\lambda+v+2)^{n-1}} \\
= & \frac{3(2-\alpha)}{4(\lambda+1)^{m}(\lambda+v+1)} \cdot \frac{(\lambda+2)^{m}(\lambda+v+2)}{(\lambda+2)^{m}(\lambda+v+2)-1} \leq 1-\alpha,
\end{aligned}
$$

and the proof is complete.

Theorem 2.4 Suppose that

$$
0 \leq \alpha \leq 1-\frac{\mathrm{T}_{1}}{\mathrm{~T}_{2}}=: \widehat{\beta}_{\max }
$$

where

$$
\begin{aligned}
\mathrm{T}_{1}:= & 8(\lambda+2)^{m}(\lambda+v+2)\left[2(\lambda+2)^{m}(\lambda+v+2)-1\right], \\
\mathrm{T}_{2}:= & 4(\lambda+1)^{m}(\lambda+v+1)\left[(\lambda+2)^{m}(\lambda+v+2)-1\right] \cdot\left[2(\lambda+2)^{m}(\lambda+v+2)-1\right] \\
& -3(\lambda+2)^{m}(\lambda+v+2) \cdot\left[2(\lambda+2)^{m}(\lambda+v+2)-1\right] \\
& -6(\lambda+2)^{m}(\lambda+v+2) \cdot\left[(\lambda+2)^{m}(\lambda+v+2)-1\right],
\end{aligned}
$$

with $\lambda>-1$ and $\nu, \mu \in \mathbb{N}$, which also satisfies

$$
\mathrm{T}_{2}>0
$$

Then, $\mathfrak{J}_{v, \lambda}^{\mu, m} \in \mathcal{K}(\alpha)$.

Proof Like in the proof of the previous theorem, if $f$ of the form (1.1) satisfies $\sum_{n=2}^{\infty}(n-$ $\alpha)\left|A_{n}\right| \leq 1-\alpha$, then $f \in \mathcal{S}^{*}(\alpha)$. From the Alexander duality relation, i.e., for $f$ of the form (1.1), we have $f \in \mathcal{K}(\alpha)$ if and only if $z f^{\prime}(z) \in \mathcal{S}^{*}(\alpha)$. Thus, according to [15, Theorem 1], if $f$ of the form (1.1) satisfies $\sum_{n=2}^{\infty} n(n-\alpha)\left|A_{n}\right| \leq 1-\alpha$, then $f \in \mathcal{K}(\alpha)$ (see also [15, Corollary on p. 110]).

Therefore, it is sufficient to prove that

$$
\mathrm{H}_{2}:=\sum_{n=2}^{\infty} n(n-\alpha)\left|\frac{(-1)^{n-1}(2 n-1)}{4^{n-1}\left[(\lambda+1)_{n-1}\right]^{m}(\lambda+\nu+1)_{(n-1) \mu}}\right| \leq 1-\alpha .
$$

Since $\lambda>-1$ and $v \in \mathbb{N}$, we have

$$
\mathrm{H}_{2}=\sum_{n=1}^{\infty} \frac{n^{2}(2 n+1)}{4^{n}\left[(\lambda+1)_{n}\right]^{m}(\lambda+v+1)_{n \mu}}+(2-\alpha) \sum_{n=1}^{\infty} \frac{n(2 n+1)}{4^{n}\left[(\lambda+1)_{n}\right]^{m}(\lambda+v+1)_{n \mu}}
$$




$$
+(1-\alpha) \sum_{n=1}^{\infty} \frac{(2 n+1)}{4^{n}\left[(\lambda+1)_{n}\right]^{m}(\lambda+v+1)_{n \mu}}
$$

and, using again inequality (2.22), we obtain

$$
\begin{aligned}
\mathrm{H}_{2} \leq & \frac{1}{4(\lambda+1)^{m}(\lambda+v+1)} \sum_{n=1}^{\infty} \frac{n^{2}(2 n+1)}{4^{n-1}\left[(\lambda+2)_{n-1}\right]^{m}(\lambda+v+2)_{n-1}} \\
& +\frac{2-\alpha}{4(\lambda+1)^{m}(\lambda+v+1)} \sum_{n=1}^{\infty} \frac{n(2 n+1)}{4^{n-1}\left[(\lambda+2)_{n-1}\right]^{m}(\lambda+v+2)_{n-1}} \\
& +\frac{1-\alpha}{4(\lambda+1)^{m}(\lambda+v+1)} \sum_{n=1}^{\infty} \frac{2 n+1}{4^{n-1}\left[(\lambda+2)_{n-1}\right]^{m}(\lambda+v+2)_{n-1}}
\end{aligned}
$$

Furthermore, a simple computation shows that the following inequalities hold:

$$
\begin{aligned}
& n^{2}(2 n+1) \leq 5 \cdot 4^{n-1}, \quad n(2 n+1) \leq 3 \cdot 4^{n-1}, \quad 2 n+1 \leq 3 \cdot 2^{n-1}, \\
& (\lambda+v+2)_{n-1} \geq(\lambda+v+2)^{n-1}, \quad(\lambda+2)_{n-1} \geq(\lambda+2)^{n-1}
\end{aligned}
$$

for all $n \in \mathbb{N}$. Using the above inequalities, together with the assumption (2.25), we get

$$
\begin{aligned}
\mathrm{H}_{2} \leq & \frac{5}{4(\lambda+1)^{m}(\lambda+v+1)} \sum_{n=1}^{\infty} \frac{1}{(\lambda+2)^{(n-1) m}(\lambda+v+2)^{n-1}} \\
& +\frac{3(2-\alpha)}{4(\lambda+1)^{m}(\lambda+v+1)} \sum_{n=1}^{\infty} \frac{1}{(\lambda+2)^{(n-1) m}(\lambda+v+2)^{n-1}} \\
& +\frac{3(1-\alpha)}{4(\lambda+1)^{m}(\lambda+v+1)} \sum_{n=1}^{\infty} \frac{1}{2^{n-1}(\lambda+2)^{(n-1) m}(\lambda+v+2)^{n-1}} \\
= & \frac{11-3 \alpha}{4(\lambda+1)^{m}(\lambda+v+1)} \cdot \frac{(\lambda+2)^{m}(\lambda+v+2)}{(\lambda+2)^{m}(\lambda+v+2)-1} \\
& +\frac{3(1-\alpha)}{4(\lambda+1)^{m}(\lambda+v+1)} \cdot \frac{2(\lambda+2)^{m}(\lambda+v+2)}{2(\lambda+2)^{m}(\lambda+v+2)-1} \\
= & \frac{(\lambda+2)^{m}(\lambda+v+2)}{4(\lambda+1)^{m}(\lambda+v+1)}\left[\frac{11-3 \alpha}{(\lambda+2)^{m}(\lambda+v+2)-1}+\frac{6(1-\alpha)}{2(\lambda+2)^{m}(\lambda+v+2)-1}\right] \\
\leq & 1-\alpha,
\end{aligned}
$$

whenever (2.24) and (2.25) hold. Thus, inequality (2.26) is satisfied and hence $\mathfrak{J}_{v, \lambda}^{\mu, m} \in$ $\mathcal{K}(\alpha)$.

Theorem 2.5 Suppose that

$$
0 \leq \alpha \leq 1-\frac{3(\lambda+2)^{m}(\lambda+v+2)}{2(\lambda+1)^{m}(\lambda+v+1)\left[2(\lambda+2)^{m}(\lambda+v+2)-1\right]},
$$

with $\lambda>-1$ and $v, \mu \in \mathbb{N}$. Then, $\frac{\mathfrak{J}_{v, \lambda}^{\mu, m}(z)}{z} \in \mathcal{P}(\alpha)$. 
Proof To obtain the required result, it is sufficient to prove that $|\mathrm{G}(z)-1|<1, z \in \mathbb{U}$, where

$$
\mathrm{G}(z):=\frac{1}{1-\alpha}\left(\frac{\mathfrak{J}_{v, \lambda}^{\mu, m}(z)}{z}-\alpha\right), \quad z \in \mathbb{U} .
$$

From the theorem of the maximum of the module of an analytic function, using the triangle inequality, inequality (2.22), and

$$
2 n+1 \leq 3 \cdot 2^{n-1}, \quad(\lambda+v+2)_{n-1} \geq(\lambda+v+2)^{n-1}, \quad(\lambda+2)_{n-1} \geq(\lambda+2)^{n-1},
$$

we get

$$
\begin{aligned}
|\mathrm{G}(z)-1| & =\left|\frac{1}{1-\alpha}\left[1+\sum_{n=1}^{\infty} \frac{(-1)^{n}(2 n+1)}{4^{n}\left[(\lambda+1)_{n}\right]^{m}(\lambda+v+1)_{n \mu}} z^{n}-\alpha\right]-1\right| \\
& =\frac{1}{1-\alpha}\left|\sum_{n=1}^{\infty} \frac{(-1)^{n}(2 n+1)}{4^{n}\left[(\lambda+1)_{n}\right]^{m}(\lambda+v+1)_{n \mu}} z^{n}\right| \\
& <\frac{1}{1-\alpha}\left|\sum_{n=1}^{\infty} \frac{(-1)^{n}(2 n+1)}{4^{n}\left[(\lambda+1)_{n}\right]^{m}(\lambda+v+1)_{n \mu}} e^{i n \theta}\right| \\
& \leq \frac{1}{1-\alpha} \sum_{n=1}^{\infty} \frac{2 n+1}{4^{n}\left[(\lambda+1)_{n}\right]^{m}(\lambda+v+1)_{n \mu}} \\
& \leq \frac{1}{4(1-\alpha)(\lambda+1)^{m}(\lambda+v+1)} \sum_{n=1}^{\infty} \frac{2 n+1}{4^{n-1}\left[(\lambda+2)_{n-1}\right]^{m}(\lambda+v+1)_{n}} \\
& \leq \frac{3}{4(1-\alpha)(\lambda+1)^{m}(\lambda+v+1)} \sum_{n=1}^{\infty} \frac{1}{2^{n-1}(\lambda+2)^{m(n-1)}(\lambda+v+2)^{n-1}} \\
& =\frac{3(\lambda+2)^{m}(\lambda+v+2)}{2(1-\alpha)(\lambda+1)^{m}(\lambda+v+1)\left[2(\lambda+2)^{m}(\lambda+v+2)-1\right]} \leq 1, \quad z \in \mathbb{U},
\end{aligned}
$$

with $\theta \in \mathbb{R}$, and the proof of our theorem is complete.

Remark 2.2 The assumptions of Theorems $2.3-2.5$ can be simultaneously fulfilled, as it is shown in Fig. 2 (the grey regions) obtained by using the MAPLE ${ }^{\mathrm{mw}}$ software.

Theorem 2.6 Suppose that $m \in \mathbb{N}, \lambda \geq \frac{\sqrt{13}-3}{2} \simeq 0.302775 \ldots$ and $v, \mu \in \mathbb{N}$. Then,

$$
\operatorname{Re}\left(\left(\mathfrak{J}_{v, \lambda}^{\mu, m}(z)\right)^{\prime} * \frac{1}{1+z}\right)>\frac{1}{2}, \quad z \in \mathbb{U}
$$

Proof We have the following power series expansion:

$$
\begin{aligned}
& \left(\mathfrak{J}_{v, \lambda}^{\mu, m}(z)\right)^{\prime} * \frac{1}{1+z}=\sum_{n=1}^{\infty} \mathrm{A}_{n} z^{n-1}, \quad z \in \mathbb{U}, \\
& \mathrm{A}_{n}=\frac{n(2 n-1)}{4^{n-1}\left[(\lambda+1)_{n-1}\right]^{m}(\lambda+v+1)_{(n-1) \mu}}, \quad n \in \mathbb{N} .
\end{aligned}
$$




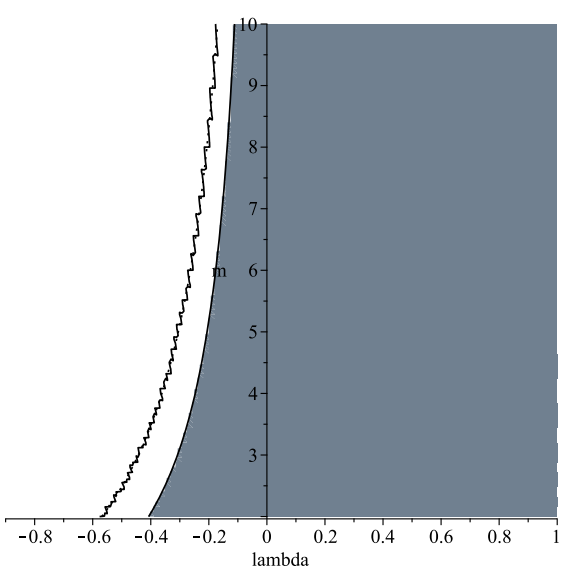

(A) The region where the assumptions (2.20) and (2.21) can be simultaneously fulfilled for $\mu=2, \nu=4$, with $\lambda \in[-0.9,1]$ and $m \in[2,10]$

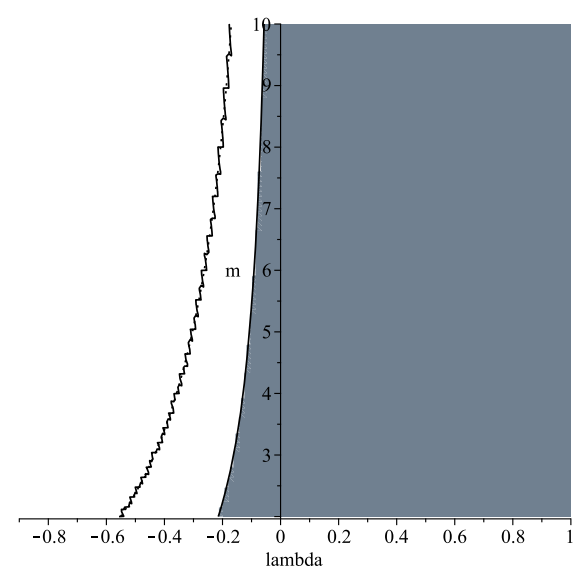

(B) The region where the assumptions (2.24) and (2.25) can be simultaneously fulfilled for $\mu=2, \nu=4$, with $\lambda \in[-0.9,1]$ and $m \in$ $[2,10]$

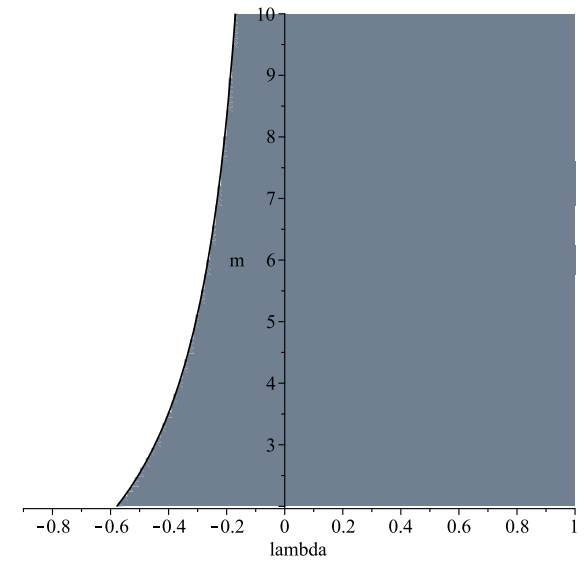

(c) The region where the assumption (2.27) is fulfilled for $\mu=2, \nu=4$, with $\lambda \in$ $[-0.9,1]$ and $m \in[2,10]$

Figure 2 The figures for Remark 2.2

We will use Lemma 1.1 to obtain the required conclusion. Thus, $\mathrm{A}_{1}=1, \mathrm{~A}_{n}>0$ for $n \geq 2$, and

$$
\mathrm{A}_{n}-\mathrm{A}_{n+1}=\frac{1}{4^{n-1}\left[(\lambda+1)_{n-1}\right]^{m}}\left[\frac{n(2 n-1)}{(\lambda+v+1)_{(n-1) \mu}}-\frac{(n+1)(2 n+1)}{4(\lambda+n)^{m}(\lambda+v+1)_{n \mu}}\right] .
$$

It is easy to check that

$$
(\lambda+v+1)_{n \mu} \geq(\lambda+v+1+(n-1) \mu)(\lambda+v+1)_{(n-1) \mu},
$$

hence

$$
\mathrm{A}_{n}-\mathrm{A}_{n+1} \geq \frac{1}{4^{n}\left[(\lambda+1)_{n-1}\right]^{m}(\lambda+v+1)_{(n-1) \mu}}
$$




$$
\begin{aligned}
& \cdot\left[4 n(2 n-1)-\frac{(n+1)(2 n+1)}{(\lambda+n)^{m}(\lambda+v+1+(n-1) \mu)}\right] \\
& =\frac{\mathrm{M}(n)}{4^{n}\left[(\lambda+1)_{n}\right]^{m}(\lambda+v+1)_{(n-1) \mu}(\lambda+v+1+(n-1) \mu)} \text {, }
\end{aligned}
$$

where

$$
\begin{aligned}
\mathrm{M}(n):= & 4 n(2 n-1)(\lambda+n)^{m}(\lambda+v+1+(n-1) \mu)-(n+1)(2 n+1) \\
= & 4 n(\lambda+n)^{m}[(\lambda+v+1)(2 n-1)+\mu(2 n-1)(n-1)] \\
& -(2 n-1)(n+2)-3, \quad n \in \mathbb{N} .
\end{aligned}
$$

First, to show that $\mathrm{A}_{n}-\mathrm{A}_{n+1} \geq 0$ for all $n \in \mathbb{N}$, we will prove that $\mathrm{M}(n) \geq 0, n \in \mathbb{N}$.

Thus, since $m \in \mathbb{N}, \lambda \geq 0$ and $v, \mu \in \mathbb{N}$, we have $\lambda+v+1+(n-1) \mu>0$, and it follows that

$$
\begin{aligned}
\mathrm{M}(n) & =(2 n-1)\left[4 n(\lambda+n)^{m}(\lambda+v+1+(n-1) \mu)-(n+2)\right]-3 \\
& \geq(2 n-1)[4 n(\lambda+n)(\lambda+v+1+(n-1) \mu)-(n+2)]-3=: \mathrm{P}(n) .
\end{aligned}
$$

For $n=1$, we get $\mathrm{P}(1)=4(\lambda+1)(\lambda+v+1)-6 \geq 4(v+1)-6>0$ because $v \in \mathbb{N}$.

For $n \geq 2$, since

$$
\mathrm{P}(n)=(2 n-1)[4 n(\lambda+n)(\lambda+v+1+(n-1) \mu)-(n+2)]-3
$$

and

$$
4(\lambda+n)(\lambda+v+1+(n-1) \mu)-(n+2) \geq 4 n(n+1)-(n+2)>0, \quad n \in \mathbb{N},
$$

it follows that

$$
\mathrm{P}(n) \geq 3[4 n(\lambda+n)(\lambda+v+1+(n-1) \mu)-(n+2)]-3:=3 \mathrm{Q}(n),
$$

where

$$
\mathrm{Q}(n):=4 n(\lambda+n)(\lambda+v+1+(n-1) \mu)-(n+3) .
$$

Under the assumptions of the theorem, we have

$$
\begin{aligned}
& 4 n(\lambda+n) \geq 4 n^{2}>n+3, \quad \text { if } n \geq 2, \\
& \lambda+v+1+(n-1) \mu>1,
\end{aligned}
$$

therefore $\mathrm{Q}(n)>0$ for all $n \geq 2$. Thus, according to inequalities (2.31) and (2.32), it follows that $\mathrm{M}(n)>0, n \in \mathbb{N}$, hence from (2.30) we conclude that $\left(\mathrm{A}_{n}\right)_{n \in \mathbb{N}}$ is a decreasing sequence.

Secondly, we need to show that

$$
\mathrm{A}_{n}-2 \mathrm{~A}_{n+1}+\mathrm{A}_{n+2} \geq 0, \quad \text { for all } n \in \mathbb{N} \text {. }
$$


Since $\mathrm{A}_{n+2}>0$ for all $n \in \mathbb{N}$, using inequality (2.29), we easily get

$$
\begin{aligned}
\mathrm{A}_{n}-2 \mathrm{~A}_{n+1}+\mathrm{A}_{n+2}> & \mathrm{A}_{n}-2 \mathrm{~A}_{n+1} \\
\geq & \frac{1}{4^{n}\left[(\lambda+1)_{n-1}\right]^{m}(\lambda+v+1)_{(n-1) \mu}} \\
& \cdot\left[4 n(2 n-1)-\frac{2(n+1)(2 n+1)}{(\lambda+n)^{m}(\lambda+v+1+(n-1) \mu)}\right] \\
= & \frac{\mathrm{L}^{*}(n)}{4^{n}\left[(\lambda+1)_{n}\right]^{m}(\lambda+v+1)_{(n-1) \mu}(\lambda+v+1+(n-1) \mu)},
\end{aligned}
$$

where

$$
\begin{aligned}
\mathrm{L}^{*}(n):= & 4 n(2 n-1)(\lambda+n)^{m}(\lambda+v+1+(n-1) \mu)-2(n+1)(2 n+1) \\
= & 4 n(\lambda+n)^{m}[(\lambda+v+1)(2 n-1)+\mu(2 n-1)(n-1)] \\
& -2(2 n-1)(n+2)-6 .
\end{aligned}
$$

Thus, to show that $\mathrm{A}_{n}-2 \mathrm{~A}_{n+1}+\mathrm{A}_{n+2} \geq 0$ for all $n \in \mathbb{N}$, it is sufficient prove that $\mathrm{L}^{*}(n) \geq 0$ for all $n \in \mathbb{N}$.

Since $m \in \mathbb{N}, \lambda \geq 0$ and $\nu, \mu \in \mathbb{N}$, we have $\lambda+v+1+(n-1) \mu>0$, and it follows that

$$
\begin{aligned}
\mathrm{L}^{*}(n) & =(2 n-1)\left[4 n(\lambda+n)^{m}(\lambda+v+1+(n-1) \mu)-2(n+2)\right]-6 \\
& \geq(2 n-1)[4 n(\lambda+n)(\lambda+v+1+(n-1) \mu)-2(n+2)]-6=: \mathrm{P}^{*}(n) .
\end{aligned}
$$

For $n=1$, we get

$$
\begin{aligned}
\mathrm{P}^{*}(1) & =4(\lambda+1)(\lambda+v+1)-12 \geq 4(\lambda+1)(\lambda+v+1)-12 \\
& \geq 4(\lambda+1)(\lambda+2)-12>0
\end{aligned}
$$

whenever $\lambda \geq \frac{\sqrt{13}-3}{2} \simeq 0.302775 \ldots$.

For $n \geq 2$, since

$$
\mathrm{P}^{*}(n)=(2 n-1)[4 n(\lambda+n)(\lambda+v+1+(n-1) \mu)-2(n+2)]-6
$$

and

$$
4(\lambda+n)(\lambda+v+1+(n-1) \mu)-2(n+2) \geq 4 n(n+1)-2(n+2)>0, \quad n \in \mathbb{N},
$$

it follows that

$$
\mathrm{P}^{*}(n) \geq 3[4 n(\lambda+n)(\lambda+v+1+(n-1) \mu)-2(n+2)]-6=: 3 \mathrm{Q}^{*}(n),
$$

where

$$
\mathrm{Q}^{*}(n):=4 n(\lambda+n)(\lambda+v+1+(n-1) \mu)-2(n+3) .
$$


Under the assumptions of the theorem, we have

$$
\begin{aligned}
& 4 n(\lambda+n) \geq 4 n^{2}>2(n+3), \quad \text { if } n \geq 2, \\
& \lambda+v+1+(n-1) \mu>1,
\end{aligned}
$$

therefore $\mathrm{Q}^{*}(n)>0$ for all $n \geq 2$. Thus, according to inequalities (2.34) and (2.35), it follows that $\mathrm{L}^{*}(n)>0, n \in \mathbb{N}$, hence from (2.33) we conclude that $\left(\mathrm{A}_{n}\right)_{n \in \mathbb{N}}$ is a convex sequence, which ends the proof.

Theorem 2.7 Suppose that $m \in \mathbb{N}, \lambda \geq \frac{\sqrt{10}-6}{4} \simeq-0.709430 \ldots$, and $v, \mu \in \mathbb{N}$. Then, $\frac{\mathfrak{J}_{v, \lambda}^{\mu, m}\left(z^{2}\right)}{z} * \sin z$ is a close-to-convex function with respect to $\frac{1}{2} \log \frac{1+z}{1-z}$.

Proof From (1.4), since

$$
\sin z=z+\sum_{n=1}^{\infty} \frac{(-1)^{n}}{(2 n+1) !} z^{2 n+1}, \quad z \in \mathbb{C}
$$

it follows that

$$
\frac{\mathfrak{J}_{v, \lambda}^{\mu, m}\left(z^{2}\right)}{z} * \sin z=z+\sum_{n=1}^{\infty} A_{2 n+1} z^{2 n+1}, \quad z \in \mathbb{U},
$$

where

$$
\mathrm{A}_{2 n+1}=\frac{2 n+1}{4^{n}\left[(\lambda+1)_{n}\right]^{m}(\lambda+v+1)_{n \mu}(2 n+1) !}, \quad n \in \mathbb{N} .
$$

In order to use Lemma 1.2, we have $\mathrm{A}_{1}=1, \mathrm{~A}_{2 n+1}>0$ for $n \geq 1$, and

$$
\begin{aligned}
(2 n & -1) \mathrm{A}_{2 n-1}-(2 n+1) \mathrm{A}_{2 n+1} \\
& =\frac{(2 n-1)^{2}}{4^{n-1}\left[(\lambda+1)_{n-1}\right]^{m}(\lambda+v+1)_{(n-1) \mu}(2 n-1) !}-\frac{(2 n+1)^{2}}{4^{n}\left[(\lambda+1)_{n}\right]^{m}(\lambda+v+1)_{n \mu}(2 n+1) !} \\
& =\frac{2 n-1}{4^{n-1}\left[(\lambda+1)_{n-1}\right]^{m}(\lambda+v+1)_{(n-1) \mu}(2 n-2) !}-\frac{2 n+1}{4^{n}\left[(\lambda+1)_{n}\right]^{m}(\lambda+v+1)_{n \mu}(2 n) !} \\
& \geq \frac{4(2 n-1)(\lambda+n)^{m}[\lambda+v+1+(n-1) \mu](2 n-1)(2 n)-(2 n+1)}{4^{n}\left[(\lambda+1)_{n}\right]^{m}(\lambda+v+1)_{n \mu}(2 n) !} \\
& =\frac{8 n(2 n-1)^{2}(\lambda+n)^{m}[\lambda+v+1+(n-1) \mu]-(2 n+1)}{4^{n}\left[(\lambda+1)_{n}\right]^{m}(\lambda+v+1)_{n \mu}(2 n) !} \\
& =\frac{\mathrm{W}(n)}{4^{n}\left[(\lambda+1)_{n}\right]^{m}(\lambda+v+1)_{n \mu}(2 n) !}, \quad n \in \mathbb{N},
\end{aligned}
$$

where

$$
\mathrm{W}(n):=8 n(2 n-1)^{2}(\lambda+n)^{m}[\lambda+v+1+(n-1) \mu]-(2 n+1), \quad n \in \mathbb{N} .
$$

It is easy to check that for $n \in \mathbb{N}$,

$$
\mathrm{W}(n):=8 n(2 n-1)^{2}(\lambda+n)^{m}[\lambda+v+1+(n-1) \mu]-(2 n+1)
$$


Table 1 Comparison of the order of starlikeness given by Theorems 2.1 and 2.3

\begin{tabular}{lllllll}
\hline$\mu$ & $v$ & $\lambda$ & $m$ & $A:=\widetilde{\alpha}_{\max }$ Theorem 2.1 & $B:=\widehat{\alpha}_{\max }$ Theorem 2.3 & $A-B \leq 0$ \\
\hline 1 & 1 & 20 & 3 & 0.99999630843873496490 & 0.99999631884528808850 & true \\
1 & 1 & 40 & 3 & 0.99999974069866645409 & 0.999999974090403376158 & true \\
1 & 1 & 75 & 3 & 0.99999997780611645794 & 0.99999997781140602240 & true \\
2 & 6 & 30 & 3 & 0.99999998209380420596 & 0.99999931958308410376 & false \\
3 & 6 & 60 & 3 & 0.99999999998948912236 & 0.99999995068295983574 & false \\
4 & 6 & 80 & 3 & 0.99999999999997698708 & 0.99999998377865092704 & false \\
\hline
\end{tabular}

Table 2 Comparison of the order of convexity given by Theorems 2.2 and 2.4

\begin{tabular}{lllllll}
\hline$\mu$ & $v$ & $\lambda$ & $m$ & $A:=\widetilde{\beta}_{\max }$ Theorem 2.2 & $B:=\widehat{\beta}_{\max }$ Theorem 2.4 & $A-B \leq 0$ \\
\hline 1 & 1 & 90 & 2 & 0.99999803110440570718 & 0.99999737481228676390 & false \\
1 & 1 & 60 & 5 & 0.99999999997135489658 & 0.99999999996180652878 & false \\
2 & 4 & 90 & 6 & 0.99999999999999971037 & 0.99999999999996292697 & false \\
2 & 4 & 60 & 2 & 0.99999990603320991858 & 0.99999173086604042377 & false \\
1 & 3 & 70 & 5 & 0.99999999998876513662 & 0.99999999998502018216 & false \\
1 & 3 & 50 & 4 & 0.99999999589402028998 & 0.99999999452536041351 & false \\
\hline
\end{tabular}

$$
\begin{aligned}
& \geq 8 n(2 n-1)^{2}(\lambda+n)[\lambda+v+1+(n-1) \mu]-(2 n+1) \\
& =(2 n-1)\{8 n(2 n-1)(\lambda+n)[\lambda+v+1+(n-1) \mu]-1\}-2=: \mathrm{W}^{*}(n) .
\end{aligned}
$$

To show that $(2 n-1) \mathrm{A}_{2 n-1}-(2 n+1) \mathrm{A}_{2 n+1} \geq 0$ for all $n \in \mathbb{N}$, we will prove that $\mathrm{W}^{*}(n) \geq 0$, $n \in \mathbb{N}$. For $n=1$, since $v \in \mathbb{N}$, we have

$$
\mathrm{W}^{*}(1)=8(\lambda+1)(\lambda+v+1)-3 \geq 8(\lambda+1)(\lambda+2)-3 \geq 0,
$$

whenever $\lambda \geq \frac{\sqrt{10}-6}{4} \simeq-0.709430 \ldots$. For $n \geq 2$, since $v, \mu \in \mathbb{N}$, we get

$$
\mathrm{W}^{*}(n) \geq 3[48(\lambda+2)(\lambda+2+\mu)-1]-2 \geq 3[48(\lambda+2)(\lambda+3)-1]-2>0,
$$

if $\lambda \geq \frac{\sqrt{10}-6}{4} \simeq-0.709430 \ldots$.

Therefore, $(2 n-1) \mathrm{A}_{2 n-1}-(2 n+1) \mathrm{A}_{2 n+1} \geq 0$ for all $n \in \mathbb{N}$, and, according to Lemma 1.2, we get our result.

Remark 2.3 1. Theorems 2.1 and 2.3 give us sufficient conditions for the starlikeness of order $\alpha$ for the function $\mathfrak{J}_{v, \lambda}^{\mu, m}$. Like we see in the above table, for some values of the parameters the first theorem gives a better result than the second, and vice versa. See Table 1.

2. For the sufficient conditions of convexity of order $\alpha$ for the function $\mathfrak{J}_{v, \lambda}^{\mu, m}$ given by Theorems 2.2 and 2.4, we cannot formulate any opinion since in the above table, for some particular values of the parameters, we obtained better results by using Theorem 2.2. See Table 2.

\section{Conclusions}

In our present investigation we have studied some geometric properties of the normalized form of the combination of generalized Lommel-Wright function, including starlikeness and convexity of order $\alpha(0 \leq \alpha<1)$, in the open unit disc using the two-sided inequality for the Fox-Wright functions that has been proved by Pogány and Srivastava in [11]. Further, we have also successfully considered conditions on $\lambda, v, \mu$, and $m$ to evaluate the 
orders of starlikeness and convexity using some classical tools. We have compared the orders of starlikeness and convexity given by both techniques to illustrate the efficacy of the approach.

\section{Acknowledgements}

The authors are grateful to the referees of this article who gave valuable comments and advice, in order to revise and improve the results of the paper in the present form.

Funding

Not applicable.

Availability of data and materials

Not applicable.

\section{Competing interests}

The authors declare that they have no competing interests.

\section{Authors' contributions}

The authors drafted and approved the final manuscript.

\section{Author details}

'Department of Mathematics and Computer Science, Faculty of Science, Menoufia University, Shebin Elkom 32511, Egypt. ${ }^{2}$ Faculty of Mathematics and Computer Science, Babeş-Bolyai University, 400084 Cluj-Napoca, Romania.

\section{Publisher's Note}

Springer Nature remains neutral with regard to jurisdictional claims in published maps and institutional affiliations.

Received: 22 June 2021 Accepted: 30 August 2021 Published online: 25 September 2021

\section{References}

1. Baricz, Á.: Bessel transforms and Hardy space of generalized Bessel functions. Mathematica 48(71)(2), 127-136 (2006)

2. Baricz, Á., Ponnusamy, S.: Starlikeness and convexity of generalized Bessel functions. Integral Transforms Spec. Funct. 21(9), 641-653 (2010)

3. de Oteiza, M.B.M., Kalla, S., Conde, S.: Un estudio sobre la función Lommel-Maitland. Rev. Téc. Fac. Ing., Univ. Zulia 9(2), 33-40 (1986)

4. Fejér, L:: Untersuchungen über Potenzreihen mit mehrfach monotoner Koeffizientenfolge. Acta Litterarum ac Scientiarum 8, 89-115 (1936)

5. Kilbas, A.A., Saigo, M., Trujillo, J.J.: On the generalized Wright function. Fract. Calc. Appl. Anal. 5(4), 437-460 (2002)

6. Miller, S.S., Mocanu, P.T.: Differential subordinates and inequalities in the complex plane. J. Differ. Equ. 67, 199-211 (1987)

7. Miller, S.S., Mocanu, P.T.: Univalence of Gaussian and confluent hypergeometric functions. Proc. Am. Math. Soc. 110(2), 333-342 (1990)

8. Orhan, H., Yağmur, N.: Geometric properties of generalized Struve functions. An. Ştiinţ. Univ. 'Al... Cuza' laşi, Mat. 63(2), 229-244 (2017)

9. Ozaki, S: On the theory of multivalent functions. Sci. Rep. Tokyo Bunrika Daigaku 2, 167-188 (1935)

10. Pathak, R.S.: Certain convergence theorems and asymptotic properties of a generalization of Lommel and Maitland transformations. Proc. Natl. Acad. Sci. India Sect. A 36(1), 81-86 (1966)

11. Pogány, T.K., Srivastava, H.M.: Some Mathieu-type series associated with the Fox-Wright function. Comput. Math. Appl. 57(1), 127-140 (2009)

12. Ponnusamy, S.: The Hardy space of hypergeometric functions. Complex Var. Theory Appl. 29(1), 83-96 (1996)

13. Ponnusamy, S., Rønning, F.: Geometric properties for convolutions of hypergeometric functions and functions with the derivative in a halfplane. Integral Transforms Spec. Funct. 8, 121-138 (1999)

14. Ruscheweyh, St:: Convolutions in Geometric Function Theory. Les Presses de 1'Université de Montréal, Montréal (1982)

15. Silverman, H.: Univalent functions with negative coefficients. Proc. Am. Math. Soc. 51(1), 109-116 (1975)

16. Yağmur, N.: Hardy space of Lommel functions. Bull. Korean Math. Soc. 52, 1035-1046 (2015)

17. Yağmur, N., Orhan, H.: Hardy space of generalized Struve functions. Complex Var. Elliptic Equ. 59(7), 929-936 (2014) 